\title{
Ecological Footprint, Public-Private Partnership Investment in Energy and Financial Development in Brazil: A Gradual Shift Causality Approach
}

\section{Gbenga Daniel Akinsola}

Girne Amerikan Üniversitesi: Girne Amerikan Universitesi

Abraham Ayobamiji Awosusi

Near East University: Yakin Dogu Universitesi

Dervis Kirikkaleli ( $\boldsymbol{\sigma}$ dkirikkaleli@eul.edu.tr)

European University of Lefke https://orcid.org/0000-0001-5733-5045

\section{Sukru Umarbeyli}

University of Mediterranean Karpasia: Akdeniz Karpaz Universitesi

Ibrahim Adeshola

Eastern Mediterranean University

\section{Tomiwa Sunday Adebayo}

Cyprus International University

\section{Research Article}

Keywords: Economic growth, ecological footprint, financial development, Public-private partnership in energy, Renewable energy consumption, Brazil

Posted Date: April 14th, 2021

DOl: https://doi.org/10.21203/rs.3.rs-328980/v1

License: (9) This work is licensed under a Creative Commons Attribution 4.0 International License. Read Full License

Version of Record: A version of this preprint was published at Environmental Science and Pollution Research on September 12th, 2021. See the published version at https://doi.org/10.1007/s11356-02115791-5. 

Ecological Footprint, Public-Private Partnership Investment in Energy and Financial Development in Brazil:
A Gradual Shift Causality Approach

Gbenga Daniel Akinsola

Department of Business Management, Faculty of Economics and Administrative Sciences, Girne American University, North Cyprus, Mersin 10-Turkey

Email: gbengadan@yahoo.com

ORCID: 0000-0003-2058-1001

Abraham Ayobamiji Awosusi

Faculty of Economics and Administrative Science, Department of Economics, Near East University, North Cyprus, Mersin 10- Turkey

Email: awosusiayobamiji@gmail.com

ORCID: 0000-0002-3533-9181

Dervis Kirikkaleli*

The European University of Lefke, Faculty of Economic and Administrative Sciences, Department of Banking and Finance, Lefke, Northern Cyprus TR-10 Mersin, Turkey

E-mail: dkirikkaleli@eul.edu.tr

ORCID: 0000-0001-5733-5045

*corresponding author

Sukru Umarbeyli

University of Mediterranean Karpasia, Northern Cyprus TR-10 Mersin Turkey ORCID: 0000-0001-7745-0606

E-mail: sukru.umarbeyli@akun.edu.tr

Ibrahim Adeshola

School of computing and technology, Department of Information Technology, Eastern Mediterranean University, Northern Cyprus, Mersin 10-Turkey

Email: Ibrahim.adeshola@emu.edu.tr

\section{Tomiwa Sunday Adebayo}

Cyprus International University, Faculty of Economic and Administrative Science, Department of Business Administration, Nicosia, Northern Cyprus, TR-10 Mersin Turkey

Email: twaikline@gmail.com

ORCID: 0000-0003-0094-1778

\section{Abstract}

The present study aims to close this gap in the literature by exploring the effect of public-private partnerships in energy and financial development on Brazil's ecological footprint by considering the impact of renewable energy and economic growth using data spanning from 1983 to 2017. The study utilized several techniques such as ARDL, FMOLS, DOLS, and CCR to examine the relationship between ecological footprint and the determinants, while the Gradual shift causality test was utilized to capture the causal linkage between the series in the presence of structural break. The outcome of the Maki Cointegration test revealed evidence of a long-run association among the variables of interest. Furthermore, the results of the ARDL, FMOLS, DOLS, and CCR tests revealed that economic growth and public and private investment in energy increase environmental degradation while both renewable energy and financial development mitigates it. Moreover, the Gradual shift causality test revealed a bidirectional causal linkage between 
ecological footprint and economic growth. The present study recommends establishing a forum that will foster public and private partnerships to enhance communication, which will create collaboration for new initiatives for green technological innovations. Additionally, the financial market can be assisted by the government by formulating a framework that would promote low carbon technology development.

53 Keywords: Economic growth; ecological footprint; financial development; Public-private partnership in energy;

54 Renewable energy consumption; Brazil

\section{Introduction}

The effect of the strain imposed by human exploitation of services and goods on nature is linked to current environmental degradation questions, economic setbacks, global warming, and ecological disruptions. In reaction, global recognition and push for sustainable growth in the context of environmental protection (sustainability) have stayed a global concern for environmentalists and economists. Increased human activities both indirectly and directly (Olanrewaju et al. 2021; Kirikaleli et al. 2020; Odugbesan and Adebayo, 2020; Rjoub et al. 2021; He et al. 2021), has increased attention paid to ecological responses from economic growth, population dynamics, energy usage, and a number of other key factors (Ayobamiji and Demet, 2020; Adedoyin et al. 2020; Kirikalelei and Adebayo, 2021; Zhang et al. 2021; Bekun et al. 2019; Adebayo, 2021). In addition to utilizing carbon dioxide $\left(\mathrm{CO}_{2}\right)$ to account for environmental quality, biocapacity and ecological footprint (EP) accounting have been introduced to include wider viewpoints. With the relevance of the Intergovernmental Panel on Climate Change (IPCC) study on "Climate change, soil erosion, habitat loss, sustainable land use, food protection and greenhouse gas(GHGs) flows in terrestrial ecosystems" (IPCC, 2017), it is clear that sustained efforts are being made to reduce pressure on global ecological capability.

Overall, given the economic growth of major economies such as Germany, the United States, India, China, and several EU nations, Japan, these influences continue to be important to the environmentalists, governments, and policymakers of these nations. In consideration of Brazil's move towards sustainable growth and environmental sustainability, appropriate strategies are being introduced towards achieving the Sustainable Development Goals (SDGs). For example, Brazil's revised 2030 climate change target contains a commitment to reduce GHGs emissions by at least $6 \%$. Brazil's long-term aim is to work on the shift of energy networks dependent on alternative energy sources and the decarbonization of the world economy by the end of the century. Brazil is one of the five big developing "BRICS" markets and the sixth-highest GHGs emission globally. Brazil has raised the intensity of its climate action in the run-up to the Paris climate change meeting.

That being said, the tumultuous developments in Brazil, such as the presidential impeachment, the pervasive economic crisis, and the massive government corruption controversy, have contributed to fears that change in climate and energy policy is losing momentum.. After incorporating EP accounting by Wackernagel and Rees (1998), the EP has continuously been utilized to determine environmental degradation. Taking into account that the EP measures the 
83 effect of human actions on the available resources of the Earth [Global Footprint Network, GFN, 2019], the analysis

84 of the complexities of the EP is set to provide more detail on the formulation of policy. The significance of mitigating

85 the effect of human activity on the environment, various studies have further investigated the variety of environmental

86 viewpoints within the EP framework (Ahmed et al., 2019; Kassouri and Altıntaş, 2020; Langnel \& Amegavi, 2020).

87 Particularly, Kassouri and Altıntaş (2020) and Langnel \& Amegavi (2020) both indorses the environmental Kuznets

88 curve (EKC) hypothesis correspondingly. The research used the EP instead of traditional CO2 as a surrogate for

89 environmental sustainability to test the EKC hypothesis's relevance. While introducing other determinants such as

90 energy consumption and financial development, both studies established an inverted U-shaped association between

91 GDP and the EP. In a wider viewpoint, the EP has been explored together with other economic indicators such as

92 financial development, renewable energy, and economic growth (Kirikaleli et al. 2020; Pata \& Yilanci, 2020; Saud et

93 al. 2020; Ahmed et al. 2021; Ahmed et al. 2019; Kassouri and Altıntaş, 2020; Langnel \& Amegavi, 2020; Ansari et

94 al. 2020). For instance, Saud et al. (2020), and Ansari et al. (2020) found a negative association between EP and GDP,

95 while Rudolph and Figge (2017), Ahmed et al. (2019), Kirikkaleli et al. (2020), and Pata \& Yilanci (2020) found

96 positive interconnection between EP and GDP. Regarding the link between EP and renewable energy, Danish \& Khan

97 (2019), Charfeddine (2017), and Yilanci \& Pata (2020) established a negative relationship between renewable energy

98 and EP. Also, the study of Kassouri and Altıntaş (2020), Godil et al. (2020), and Ahmed et al. (2021) established a

99 positive relationship between EP and financial development, while the study of Ahmed et al. (2019) and Kirikaleli

100 and Adebayo, (2020) established a negative relationship between financial development and EP.

101 Questions still stayed about the role of public-private investment in energy, GDP growth, renewable energy use,

102 and financial development in mitigating climate change. Nevertheless, the complexity of these variables due to the

103 varying economic system and environmental policies across nations makes it extremely difficult to agree on climate

104 change determinants across countries. The empirical evidence provided in this report is therefore helpful in

105 formulating policies relating to climate change. The above motives point to the objective of studying the dynamic

106 effect of public-private investment in energy, financial development, economic growth and renewable energy on the

107 EP in Brazil utilizing current econometric techniques. In advancing the study of Alola et al. (2019) and Kirikaleli et

108 al. (2020), the study utilized yearly data between 1984 and 2017. In addition to the ongoing research, the paper

109 considered the utilization of EP against regular CO2 because Brazil is already striving to deal with the ecological

110 deficit issues (Global Footprint Network, GFN, 2019). Also, we incorporate public-private investment in energy in 111 the framework - to assess its impact on the EP

112 The study's remaining sections are ordered as follows: Section 2 presents the summary of past studies, and section

1133 illustrates the data and methodology. This is followed by an analysis of data analysis and discussion in section 4 and 114 the conclusion and policy path are presented in section 5.

115

116 2. Literature Review 
This research examines the interaction between renewable energy and GDP on EP by considering the role of 118 financial development and public-private partnership investment in energy (PPI) in Brazil. In China, Khan et al. (2020) 119 explore the effects of PPI on environmental degradation using quarterly data ranging from 1990Q1 to 2017Q1. The 120 empirical result shows that PPI exerts a detrimental effect on environmental quality. Using the BARDL (bootstrapping 121 autoregressive distributed lag modeling), Shahbaz et al. (2020) also reveal a similar outcome in China. In India, 122 Kirikkaleli \& Adebayo (2021) also examine the interaction between PPI and environmental degradation. The study's 123 outcome reveals that the interaction between PPI and environmental deterioration is positive. Shahbaz et al. (2018) 124 also explore the association between PPI and environmental degradation for France by using research \& development 125 to indicate public investment in energy (PI). The result shows a negative connection between PPI and environmental 126 degradation in France. Furthermore, a one-way causal association from PI to environmental degradation. Waqih et al. 127 (2019) also followed a similar concept using foreign direct investment as a metric for private investment for the 128 SAARC region. The panel data was utilized spanning from 1986 to 2014, using the PARDL and PFMOLS. Results 129 show the private investment has a positive effect on the environmental deterioration in the SAARC region. Studies 130 incorporating the PPI into the environmental literature are limited.

131 In Turkey, Godil et al. (2020) explore the interaction between ecological footprint and financial development 132 utilizing data ranging from 1986 to 2018 . The empirical finding shows financial development influences on ecological 133 footprint is positive. Sabir and Gorus (2019) found that a positive association between ecological footprint and GDP 134 between 1975 and 2017 in South Asian countries. Kirikkaleli et al. (2020) employed the dual adjustment approach to 135 scrutinize GDP's impact on the ecological footprint for 32 years (1985-2017). Results show that economic growth 136 influence ecological footprint positively. In G7 countries, Pata \& Yilanci (2020) scrutinized the interconnection 137 between financial development and economic growth on ecological footprint covering 1980 to 2015 . The authors 138 assert that financial development will be an effective tool to regulate ecological footprint in three countries amongst 139 G7 countries. However, economic growth contributes to ecological footprint. Wang et al. (2020) explore the 140 interconnection between GDP and ecological footprint from 1980 to 2016. Results show that economic growth tends 141 to induce ecological footprint but a one-way causality flowing from GDP to ecological footprint. Saud et al. (2020) 142 explored the effect of economic growth and financial development on ecological footprint, covering the period from 1431990 to 2014. The result indicates that financial development and GDP have a feedback causal connection to 144 ecological footprint. Ahmed et al. (2021) found that economic growth and financial development positively impact 145 Japan's ecological footprint between 1971 and 2016.

146 Rudolph and Figge (2017) established economic growth positively influence ecological footprint in 146 147 nations between 1981 and 2009. Ahmed et al. (2019) examined the link between economic growth and ecological 148 footprint in Malaysia between 1971 and 2014, while the empirical result shows that economic growth tends to induce 149 ecological footprint, but financial development mitigated it. Kassouri and Altıntaş (2020) explored the association 150 between ecological footprint and financial development in 13 MENA nations covering the period between 1990 and 151 2016. There exist a positive association between ecological footprint and financial development. Langnel \& Amegavi 152 (2020) found that economic growth is positively affected by Ghana's ecological footprint and the Granger causality 
153 test revealed a two-way causal connection between economic growth and ecological footprint. Ansari et al. (2020)

154 investigated the effect of economic growth on the ecological footprint in the Gulf Cooperation Council nations 155 between 1991 and 2017, but the empirical outcomes show that economic growth stimulates ecological footprint.

156 Hassan et al. (2019) scrutinized the connection between economic growth and ecological footprint in Pakistan between

1571971 and 2014. The empirical evidence shows a positive interconnection and no causal link between ecological 158 footprint and GDP. Ibrahiem \& Hanafy (2020) probed into the interconnection of economic growth and ecological 159 footprint in Egypt covering the time-period 1971 to 2014 employing FMOLS, DOLS, and Toda-Yamamoto approach.

160 Results reveal that economic growth positively impacts ecological footprint.

Furthermore, GDP granger causes ecological footprints. Udemba (2020) employed the ARDL method, which

162 shows a positive connection between GDP and ecological footprints spanning between 1981 and 2018 in Nigeria, but 163 the causality test shows a one-way causal interconnection from economic growth to ecological footprints. Alola et al.

164 (2019) explore the connection of renewable energy use and GDP on ecological footprints for 16 EU nations. The 165 effects of renewable energy and economic growth on ecological footprint show to be positive and negative, 166 respectively. Usman et al. (2020b) examined the interconnection between economic growth and ecological footprints 167 spanning between 1971 and 2014 in Brazil and using the FMOLS and DOLS in capturing long-run association while 168 the VECM to establish the causal link. FMOLS and DOLS outcomes show that economic growth and ecological 169 footprints are positively related, but there is no causal link between ecological footprints and economic growth. 170 Nathaniel \& Khan (2020) scrutinized the role of economic growth and renewable energy on the ecological footprint 171 in ASEAN countries, using data ranging between 1990 and 2016. The empirical outcome shows that economic growth 172 increases ecological footprints; renewable energy contributes to improving the environment's quality. Moreover, a 173 feedback causality exists between ecological footprints and renewable energy.

174 Usman et al. (2021) used the 15 largest emitting nation's panel data covering the period 1990 to 2017 to 175 probe into the connection between renewable energy, GDP, and financial development on ecological footprint. The 176 PARDL method's outcome shows financial development and renewable energy contributes to environmental quality 177 while GDP contributes to environmental degradation. The Granger causality test reveals a feedback causal 178 interconnection between ecological footprint and these variables (renewable energy, financial development, and 179 GDP). Utilizing the quarterly data spanning from 1985Q1 to 2014Q4, Usman et al. (2020a) explored the effect of 180 renewable energy, GDP, and financial development using the ARDL method. The empirical outcome suggests that 181 renewable energy and GDP negatively impact ecological footprint, but the financial development positively affects 182 environmental degradation and a one-way causal interconnection from ecological footprint to financial development 183 and real output. Silence \& Pata (2020) inspected the association between GDP and ecological footprint in China 184 between 1965 and 2016 while the empirical results reported a positive link between ecological footprint and GDP.

185 Destek et al. (2018) establish a U-shaped interconnection between GDP and ecological footprint while renewable 186 energy increases the environment's quality in EU countries between 1980 and 2013. Danish \& Khan (2019) examined 187 the linkage between renewable energy and GDP on ecological footprint using the panel data covering 1992 to 2016 188 for BRICS economies while the empirical results reveal that renewable energy decreases environmental deterioration, 

scrutinized the interconnection between financial development and economic growth on Qatar's ecological footprint, spanning between 1970 and 2015. The empirical results indicate that financial development negatively relates to ecological footprint but economic growth positively relates to environmental degradation.

193

\begin{tabular}{|c|c|c|c|c|c|}
\hline Investigator (s) & Timeframe & Nation (s) & Variable used & Technique(s) & Findings \\
\hline Godil et al. (2020) & 1986 to 2018 & China & $\begin{array}{l}\text { EP, TU. GLO, and } \\
\text { FD }\end{array}$ & QARDL & $\begin{array}{c}\mathrm{FD} \rightarrow \mathrm{EP}(+) \\
\mathrm{TU} \rightarrow \mathrm{EP}(+) \\
\mathrm{GLO} \rightarrow \mathrm{EP}(+)\end{array}$ \\
\hline $\begin{array}{l}\text { Kirikkaleli, et al. } \\
\text { (2020) }\end{array}$ & 1985 to 2017 & Turkey & $\begin{array}{l}\text { EP, TR, EC, GLO, } \\
\text { and GDP }\end{array}$ & $\begin{array}{l}\text { dual adjustment } \\
\text { approach }\end{array}$ & $\begin{array}{c}\mathrm{GDP} \rightarrow \mathrm{EP}(+) \\
\mathrm{GLO} \rightarrow \mathrm{EP}(+) \\
\mathrm{TR} \rightarrow \mathrm{EP}(+) \\
\mathrm{EC} \rightarrow \mathrm{EP}(+)\end{array}$ \\
\hline Pata \& Yilanci (2020) & 1980 to 2015 & $\begin{array}{c}\mathrm{G} 7 \\
\text { countries }\end{array}$ & $\begin{array}{c}\mathrm{EP}, \mathrm{FD}, \mathrm{EC}, \mathrm{GLO}, \\
\text { GDP }\end{array}$ & $\begin{array}{c}\text { Fourier T-Y } \\
\text { causality }\end{array}$ & $\begin{array}{c}\mathrm{GDP} \rightarrow \mathrm{EP}(+) \\
\mathrm{GLO} \rightarrow \mathrm{EP} \\
\mathrm{EC} \rightarrow \mathrm{EP} \\
\mathrm{FD} \rightarrow \mathrm{EP}\end{array}$ \\
\hline Saud et al. (2020) & 1990 to 2014 & $\begin{array}{l}\text { OBORI } \\
\text { nations }\end{array}$ & $\begin{array}{l}\text { EP, FD, TR, EC, } \\
\text { GLO, GDP }\end{array}$ & $\begin{array}{c}\text { PARDL, DH } \\
\text { Granger causality }\end{array}$ & $\begin{array}{c}\mathrm{FD} \leftrightarrow \mathrm{EP} \\
\mathrm{TR} \leftrightarrow \mathrm{EP} \\
\mathrm{TR} \leftrightarrow \mathrm{GDP} \\
\mathrm{GDP} \rightarrow \mathrm{EP}(-)\end{array}$ \\
\hline Ahmed et al. (2021) & 1971 to 2016 & Japan & $\begin{array}{c}\text { EP, FD, POP, EC, } \\
\text { GLO, GDP }\end{array}$ & Non-linear ARDL & $\mathrm{FD}^{+} \rightarrow \mathrm{EP}(+)$ \\
\hline $\begin{array}{l}\text { Rudolph and Figge } \\
\text { (2017) }\end{array}$ & 1981 to 2009 & 146 nations & EP, GDP, GLO & Driscoll and Kraay & $\begin{array}{c}\mathrm{GDP} \rightarrow \mathrm{EP}(+) \\
\mathrm{GLO} \neq \mathrm{EP}\end{array}$ \\
\hline Ahmed et al. (2019) & 1971 to 2014 & Malaysia & $\begin{array}{l}\text { EP, POP, EC, } \\
\text { GLO, GDP }\end{array}$ & $\begin{array}{c}\text { Bayer \& Hanck co- } \\
\text { integration test and } \\
\text { ARDL }\end{array}$ & $\begin{array}{c}\mathrm{GDP} \rightarrow \mathrm{EP}(+) \\
\mathrm{POP} \rightarrow \mathrm{EP}(+) \\
\mathrm{EC} \rightarrow \mathrm{EP}(+) \\
\mathrm{FD} \rightarrow \mathrm{EP}(-) \\
\mathrm{GLO} \neq \mathrm{EP}\end{array}$ \\
\hline $\begin{array}{l}\text { Kassouri and Altıntaş } \\
(2020)\end{array}$ & 1990 to 2016 & $\begin{array}{l}13 \text { MENA } \\
\text { nations }\end{array}$ & $\begin{array}{l}\text { EP, FD, HD, OIL, } \\
\text { URB, GLO, GDP }\end{array}$ & IFE and CCEMG & $\begin{array}{l}\mathrm{FD} \rightarrow \mathrm{EP}(+) \\
\mathrm{OIL} \rightarrow \mathrm{EP}(+) \\
\mathrm{HD} \rightarrow \mathrm{EP}(+) \\
\mathrm{URB} \rightarrow \mathrm{EP}(-)\end{array}$ \\
\hline $\begin{array}{l}\text { Langnel \& Amegavi } \\
\qquad(2020\end{array}$ & 1971 to 2016 & Ghana & $\begin{array}{l}\text { EP, GLO, EC, } \\
\text { Urban }\end{array}$ & ARDL & $\begin{array}{c}\mathrm{EC} \leftrightarrow \mathrm{EP} \\
\mathrm{GLO} \rightarrow \mathrm{EP} \\
\mathrm{URB} \rightarrow \mathrm{EP}(+) \\
\mathrm{GDP} \rightarrow \mathrm{EP}(+)\end{array}$ \\
\hline Ansari et al. (2020) & 1991 to 2017 & $\begin{array}{l}\text { GCC } \\
\text { nations }\end{array}$ & $\begin{array}{l}\text { EP, GLO, EC, } \\
\text { GDP }\end{array}$ & $\begin{array}{l}\text { FMOLS, DOLS, } \\
\text { westerlund } \\
\text { cointegration }\end{array}$ & $\begin{array}{c}\mathrm{GDP} \rightarrow \mathrm{EP}(-) \\
\mathrm{EC} \rightarrow \mathrm{EP}(+) \\
\mathrm{GLO} \rightarrow \mathrm{EP}(+)\end{array}$ \\
\hline Wang et al. (2020) & 1980 to 2016 & $\begin{array}{c}\mathrm{G} 7 \\
\text { countries }\end{array}$ & $\begin{array}{l}\text { EP, NR, GLO, } \\
\text { BIO, GDP }\end{array}$ & $\begin{array}{l}\text { bootstrap panel co- } \\
\text { integration, DSUR } \\
\text { estimator, D-H } \\
\text { Granger causality } \\
\text { test } \\
\end{array}$ & $\begin{array}{l}\mathrm{GDP} \rightarrow \mathrm{EP}(+) \\
\mathrm{GLO} \rightarrow \mathrm{EP}(-) \\
\mathrm{BIO} \rightarrow \mathrm{EP}(+) \\
\mathrm{NR} \rightarrow \mathrm{EP}(+)\end{array}$ \\
\hline Hassan et al. (2019) & 1971 to 2014 & Pakistan & $\begin{array}{c}\text { EP, GDP, HD, } \\
\text { URB }\end{array}$ & $\begin{array}{l}\text { Bayer-Hanck co- } \\
\text { integration, ARDL }\end{array}$ & $\begin{array}{c}\mathrm{GDP} \rightarrow \mathrm{EP}(+) \\
\mathrm{URB} \neq \mathrm{EP}(-) \\
\mathrm{HD} \neq \mathrm{EP}(-)\end{array}$ \\
\hline
\end{tabular}




\begin{tabular}{|c|c|c|c|c|c|}
\hline $\begin{array}{l}\text { Ibrahiem \& Hanafy } \\
(2020)\end{array}$ & 1971 to 2014 & Egypt & $\begin{array}{l}\text { EP, POP, GLO, } \\
\text { EC, GDP }\end{array}$ & $\begin{array}{l}\text { FMOLS, DOLS and } \\
\text { T-Y approach }\end{array}$ & $\begin{array}{l}\mathrm{GDP} \leftrightarrow \mathrm{EP}(+) \\
\mathrm{GLO} \rightarrow \mathrm{POP} \\
\mathrm{GLO} \rightarrow \mathrm{EC} \\
\mathrm{POP} \rightarrow \mathrm{EP} \\
\mathrm{POP} \rightarrow \mathrm{GDP}\end{array}$ \\
\hline Udemba (2020) & 1981 to 2018 & Nigeria & $\begin{array}{l}\text { EP, GDP, Agric, } \\
\text { FDI, EC, POP }\end{array}$ & ARDL & $\begin{array}{c}\mathrm{GDP} \rightarrow \mathrm{EP}(+) \\
\mathrm{POP} \rightarrow \mathrm{EP}(-) \\
\text { Agric } \rightarrow \mathrm{EP}(+) \\
\mathrm{EC} \rightarrow \mathrm{EP}(+) \\
\mathrm{FDI} \rightarrow \mathrm{EP}(+)\end{array}$ \\
\hline Alola et al. (2019) & 1997 to 2014 & $\begin{array}{l}16 \mathrm{EU} \\
\text { nations }\end{array}$ & $\begin{array}{l}\text { EP, REN, EC, } \\
\text { GDP, TR, FR }\end{array}$ & $\begin{array}{l}\text { PMG-ARDL, D-H } \\
\text { Granger Causality }\end{array}$ & $\begin{aligned} & \mathrm{GDP} \rightarrow \mathrm{EP}(+) \\
& \mathrm{REN} \leftrightarrow \mathrm{EP}(+) \\
& \mathrm{TR} \rightarrow \mathrm{EP}(-) \\
& \mathrm{FR} \leftrightarrow \mathrm{EP}(-) \\
& \mathrm{EC} \rightarrow \mathrm{EP}(+)\end{aligned}$ \\
\hline Usman et al. (2020b) & 1971 to 2014 & Brazil & $\begin{array}{l}\text { EP, EC, Demo, } \\
\text { GDP, GLO }\end{array}$ & $\begin{array}{l}\text { FMOLS, DOLS, } \\
\text { VECM Granger } \\
\text { Causality }\end{array}$ & $\mathrm{GDP} \rightarrow \mathrm{EP}(+)$ \\
\hline $\begin{array}{l}\text { Nathaniel \& Khan } \\
\text { (2020) }\end{array}$ & 1990 to 2016 & $\begin{array}{l}\text { ASEAN } \\
\text { countries }\end{array}$ & $\begin{array}{l}\text { EP, GDP, REN, } \\
\text { TR, URB, EC }\end{array}$ & $\begin{array}{l}\text { PMG-ARDL, D-H } \\
\text { Granger Causality }\end{array}$ & $\begin{array}{l}\mathrm{GDP} \rightarrow \mathrm{EP}(+) \\
\mathrm{REN} \leftrightarrow \mathrm{EP}(-) \\
\mathrm{TR} \rightarrow \mathrm{EP}(+) \\
\mathrm{EC} \rightarrow \mathrm{EP}(+) \\
\mathrm{URB} \rightarrow \mathrm{EC}\end{array}$ \\
\hline Usman et al. (2021) & 1990 to 2017 & $\begin{array}{l}15 \text { large } \\
\text { emitting } \\
\text { nation }\end{array}$ & $\begin{array}{l}\text { EP, FD, REN, } \\
\text { GDP }\end{array}$ & PARDL & $\begin{array}{c}\mathrm{GDP} \rightarrow \mathrm{EP}(+) \\
\mathrm{REN} \rightarrow \mathrm{EP}(-) \\
\mathrm{FD} \rightarrow \mathrm{EP}(-)\end{array}$ \\
\hline Usman et al. (2020a) & $\begin{array}{l}\text { 1985Q1 to } \\
\text { 2014Q4 }\end{array}$ & USA & $\begin{array}{l}\text { EP, REN, GLO, } \\
\text { FD, GDP }\end{array}$ & $\begin{array}{c}\text { ARDL, VECM } \\
\text { Granger causality } \\
\text { test }\end{array}$ & $\begin{array}{c}\mathrm{GDP} \rightarrow \mathrm{EP}(-) \\
\mathrm{FD} \rightarrow \mathrm{EP}(+) \\
\mathrm{REN} \rightarrow \mathrm{EP}(-) \\
\mathrm{GLO} \rightarrow \mathrm{EP}(+)\end{array}$ \\
\hline Yilanci \& Pata (2020) & 1965 to 2016 & China & EP, EC, GDP & Fourier ARDL & $\begin{array}{c}\mathrm{GDP} \rightarrow \mathrm{EP}(+) \\
\mathrm{EC} \rightarrow \mathrm{EP}(-)\end{array}$ \\
\hline Destek et al. (2018) & 1980 to 2013 & $\begin{array}{c}\text { EU } \\
\text { countries }\end{array}$ & $\begin{array}{l}\text { EP, GDP, REN, } \\
\text { EC, TR }\end{array}$ & $\begin{array}{l}\text { PMG-ARDL, } \\
\text { FMOLS, DOLS, }\end{array}$ & $\begin{array}{c}\mathrm{GDP} \rightarrow \mathrm{EP}(-) \\
\mathrm{REN} \rightarrow \mathrm{EP}(-) \\
\mathrm{TR} \rightarrow \mathrm{EP}(-) \\
\mathrm{EC} \rightarrow \mathrm{EP}(+)\end{array}$ \\
\hline Danish \& Khan (2019) & 1992 to 2016 & $\begin{array}{l}\text { BRICS } \\
\text { economies }\end{array}$ & $\begin{array}{l}\text { EP, URB, REN, } \\
\text { NR, GDP }\end{array}$ & $\begin{array}{l}\text { PMG-ARDL, } \\
\text { FMOLS, DOLS }\end{array}$ & $\begin{array}{c}\mathrm{GDP} \leftrightarrow \mathrm{EP}(+) \\
\mathrm{REN} \leftrightarrow \mathrm{EP}(-) \\
\mathrm{NR} \leftrightarrow \mathrm{EP} \\
\mathrm{URB} \leftrightarrow \mathrm{EP}\end{array}$ \\
\hline Charfeddine (2017) & 1970 to 2015 & Qatar & $\begin{array}{l}\text { Markov Switching } \\
\text { model }\end{array}$ & $\begin{array}{c}\text { EP, FD, GDP, EC, } \\
\text { TR, }\end{array}$ & $\begin{array}{c}\mathrm{GDP} \rightarrow \mathrm{EP}(+) \\
\mathrm{FD} \rightarrow \mathrm{EP}(-) \\
\mathrm{EC} \rightarrow \mathrm{EP}(+)\end{array}$ \\
\hline \multicolumn{6}{|c|}{$\begin{array}{l}\text { Note: EC: Energy Consumption, GDP: Economic Growth, EP: ecological footprint, } \rightarrow(+) \text { : Positive relationship, } \rightarrow(-) \\
\text { Negative relationship, } \rightarrow \text { : One-way causality, } \leftrightarrow \text { : Bidirectional causality, URB: Urbanization, TR: Trade openness, TU } \\
\text { Tourism, GLO: globalization, POLS: Pooled Ordinary Least Squares, IFE: Interactive Fixed Effects, RE: Random Effect, T } \\
\text { Y: Toda-Yamamoto causality, POP: population density, CCEMG: Common Correlated Effects Mean Group, OIL: oil rents } \\
\text { HD: Human development, NR: Natural resources, BIO: Biomass, Agric: Agriculture, FR: fertility rate }\end{array}$} \\
\hline
\end{tabular}
194 


\section{Data, theoretical rationale and methodology}

a. Data

In this research, the long-run and casual effect of economic growth, renewable energy, public-private partnership investment in energy and financial development on ecological footprints in Brazil was explored. This study utilized the times series data spanning between 1984 and 2017. The study sourced the ecological footprints from Global Footprint Network (GFN, 2021) database, public-private partnership investment in energy and economic growth were derived from the World Bank database (World Bank, 2020); the renewable energy use was sourced from the British petroleum database whereas the financial development index was obtained from the International monetary fund (IMF, 2019). The description, unit of measurement and source of the variable used was presented in Table 1. The variables' natural log was taken to address the heteroscedasticity problems, reduce skewness, and reveal the outcome as elasticities. Equations 1 and 2 are the economic and econometric models, respectively, guarding this study.

As mentioned earlier, EF denoted ecological footprints, GDP denotes economic growth, FD, REN, and PPI denote financial development index, renewable energy use, and public-private partnership investment in energy correspondingly. Parameters of these variables were denoted as $\beta_{0}, \beta_{1}, \beta_{2}, \beta_{3}$, and $\beta_{4}$. The subscript of $t$ denotes the period (1984-2017), while the residual error of the model was represented as $\varepsilon$.

\begin{tabular}{|c|c|c|c|}
\hline \multicolumn{4}{|l|}{ Table 1: Units and Sources of the Variables } \\
\hline Code & Description & Unit of measurement & Sources \\
\hline EP & Ecological footprints & Global hectare of land & $\begin{array}{c}\text { Global Footprint Network } \\
\text { (GFN, 2021) }\end{array}$ \\
\hline PPI & $\begin{array}{c}\text { Public-private partnership } \\
\text { investment in energy }\end{array}$ & current US\$ & World Bank, (2021) \\
\hline GDP & Economic growth & $\begin{array}{c}\text { GDP per Capita Constant } \\
\text { US\$, 2010 }\end{array}$ & \\
\hline REN & Renewable energy & $\begin{array}{c}\text { Renewables per capita } \\
(\mathrm{kWh})\end{array}$ & BP (2021) \\
\hline FD & Financial development & Index & IMF (2021) \\
\hline
\end{tabular}

$$
E P_{t}=f\left(G D P_{t}, P P I_{t}, R E N_{t} F D_{t}\right)
$$

$$
E P_{t}=\beta_{0}+\beta_{1} G D P_{t}+\beta_{2} P P I_{t}+\beta_{3} R E N_{t}+\beta_{4} F D_{t}+\varepsilon_{t}
$$




\section{b. Theoretical rationale}

217 The ecological footprint is a distinctive metric of environmental quality in other natural areas required to promote 218 economic growth. The availability of water resources, forest reserves, agricultural or grazing land, and fresh air is part 219 of the natural areas derived through ecological footprints. These natural areas' capacity depends greatly on the 220 eutrophication potential, terrestrial acidification, and ecotoxicity of the ecosystem and the environment. The ecological 221 footprint is a more comprehensive proxy for measuring environmental degradation because it consists of $222 \mathrm{CO}_{2}$ emission, grazing land, fishing grounds, cropland, and forest products, compared to previous literature that 223 utilizes only $\mathrm{CO}_{2}$ emission. On this premise, the ecological footprint was adopted to measure environmental 224 degradation. Ecological footprints provide a bigger definition of environmental quality compared to $\mathrm{CO}_{2}$ emission 225 that is arguably flawed. To improve the environment literature, the impact of economic growth, renewable energy, 226 financial development, and public-private partnership investment in energy on Brazil's environmental quality. An 227 increase in the growth of the economy, occasion the rise of energy demand, which contributes to environmental 228 deterioration (Charfeddine, L., \& Mrabet, Z. (2017); Wang \& Dong (2019); Bello et al.(2018); Destek \& Sinha (2020); 229 Ahmed et al. (2020); Hassan, et al. 2019; Kirikkaleli et al. 2020). Moreover, economic expansion and ecological 230 footprint are positively related. Therefore, GDP is expected to cause an increase in environmental deterioration $\left(\beta_{1}=\right.$

$\left.231 \frac{\delta E P}{\delta G D P}>0\right)$. Following the study of Khan et al. (2020), and Kirikkaleli \& Adebayo (2021). This study incorporates 232 public-private partnership investment in energy to this study's model. We anticipate that there will be a negative 233 association between ecological footprint and public-private partnership investment in energy. Thus, public-private 234 partnership investment in energy should reduce environmental degradation. $\left(\beta_{2}=\frac{\partial E P}{\partial P P I}<0\right)$, or $\left(\beta_{2}=\frac{\partial E P}{\partial P P I}>0\right)$ if 235 PPI is not eco-friendly. Renewable energy use was incorporated into the framework, which is in consonance with 236 Murshed et al. (2021), Wang \& Dong (2019), and Usman, et al. (2021). The interaction between renewable energy 237 and ecological footprint is expected to be negative $\left(\beta_{3}=\frac{\delta E P}{\delta R E N}<0\right)$. Thus, environment quality will be improved by 238 renewable energy use. Following the study of Destek \& Sarkodie (2019), Naqvi, et al. (2020), Chen et al. (2019), 239 Usman \& Hammar (2020) and Yao et al. (2021, we added financial development to our empirical model. It is 240 anticipated that the interaction between financial development and ecological footprints is negative $\left(\beta_{4}=\frac{\delta E P}{\delta F D}<0\right)$.

\section{1 c. Methodology}

\section{Stationarity Tests}

ADF and PP unit root tests developed by Said and Dickey (1984) and Phillips \& Perron (1988) were used to

244 determine the variable's stationarity properties. As stated by Adebayo \& Akinsola (2021) and Khan et al. (2021), the 245 conventional unit-roots outcome is usually inconsistent because they do not put into consideration the structural 246 break(s) during estimation. For this purpose, the study used the Zivot and Andrews (2002) unit root test, which can 247 detect at least one structural break in the series, which was defined as follows: 
248 Model A: $\Delta \mathrm{y}=\sigma+\hat{\mathrm{u}} y_{t-1}+\beta \mathrm{t}+\gamma \mathrm{DU} t+\sum_{j=i}^{t} d_{j} \Delta y_{t-j}+\varepsilon_{t}$

249 Model B: $\Delta \mathrm{y}=\sigma+\hat{\mathrm{u}} y_{t-1}+\beta \mathrm{t}+\theta_{\mathrm{DT}}+\sum_{j=i}^{t} d_{j} \Delta y_{t-j}+\varepsilon_{t}$

250 Model C: $\Delta \mathrm{y}=\sigma+\hat{\mathrm{u}} y_{t-1}+\beta \mathrm{t}+\theta_{\mathrm{DT}} \gamma \mathrm{DU}_{t}+\sum_{j=i}^{t} d_{j} \Delta y_{t-j}+\varepsilon_{t}$

251 Where; $\mathrm{DU}_{\mathrm{t}}$ denotes the mean shift of the dummy variable, which occurs at possible break-date (TB); $\mathrm{DT}_{\mathrm{t}}$ denotes 252 the trend shift of the corresponding variable used. Formally,

253

$\mathrm{DU}_{\mathrm{t}}=\left\{\begin{array}{l}1 \ldots \ldots \ldots \text { if } t>T B \\ 0 \ldots \ldots \ldots \text { otherwise }\end{array}\right.$ and $\mathrm{DU}_{\mathrm{t}}=\left\{\begin{array}{l}t-T B \ldots \ldots \text { if } t>T B \\ 0 \ldots \ldots \ldots \text { therwise }\end{array}\right.$

\section{Maki Cointegration}

255 This study employed Maki co-integration test examined the long-run association amongst the variables of interest. 256 However, one of the uniqueness of this technique is capturing at least five structural breaks simultaneously. The four 257 regression models recommended by Maki (2012) are stated as follows;

258 Level shift;

$$
Y_{t}=\rho+\sum_{i=1}^{k} \rho_{i} D_{i, t}+\theta^{\imath} Z_{t}+\varepsilon_{t}
$$

Level shift with trend

$$
Y_{t}=\rho+\sum_{i=1}^{k} \rho_{i} D_{i, t}+\theta^{\imath} Z_{t}+\sum_{i=1}^{k} \theta^{\imath} Z_{t} D_{i, t}+\varepsilon_{t}
$$

262 Regime shifts

$$
Y_{t}=\rho+\sum_{i=1}^{k} \rho_{i} D_{i, t}+\theta^{\imath} Z_{t}+\sigma t+\sum_{i=1}^{k} \theta^{\imath} Z_{t} D_{i, t}+\varepsilon_{t}
$$

Trend and Regime shifts

$$
Y_{t}=\rho+\sum_{i=1}^{k} \rho_{i} D_{i, t}+\theta^{\imath} Z_{t}+\sigma t+\sum_{i=1}^{k} \sigma^{\iota} D_{i, t}+\sum_{i=1}^{k} \theta^{\iota} Z_{t} D_{i, t}+\varepsilon_{t}
$$

where: $Y_{t}$. depicts $E F$ and $Z_{t}$ depicts its regressors

267 In testing for the long-run association amongst the variables of interest, the ARDL (autoregressive distributive lag)

268 bounds testing approach initiated by Pesaran et al. (2001) was utilized as a robustness test for the Maki co-integration

269 test. This technique has the following advantages over the conventional co-integration methods in the following ways:

270 (i) It accommodates variables with different integrated order; (ii) Endogeneity and serial correlation problem are 
addressed by selecting the appropriate lag length; (iii) It produces accurate estimation for small sample size; (iv) It also allows the short and long-run estimates concurrently. These advantages make ARDL the most suitable econometric technique for this study. ARDL model was defined as follows;

$$
\begin{aligned}
\Delta E P_{t}=\theta_{0}+\sum_{i=1}^{t} & \theta_{1} \Delta E P_{t-i}+\sum_{i=1}^{t} \theta_{2} \Delta P P I_{t-i}+\sum_{i=1}^{t} \theta_{3} \Delta G D P_{t-i}+\sum_{i=1}^{t} \theta_{4} \Delta R E N_{t-i}+\sum_{i=1}^{t} \theta_{5} \Delta F D_{t-i}+\beta_{1} E P_{t-1} \\
& +\beta_{2} P P I_{t-1}+\beta_{3} G D P_{t-1}+\beta_{4} R E N_{t-1}+\beta_{5} F D_{t-1}+E C T_{t-1} \\
& +\varepsilon_{t}
\end{aligned}
$$
hypotheses $\left(H_{a}\right)$ denotes the presence of co-integration, which is illustrated in Equations 12 and 13. When the lower and upper bond critical values is greater than the F-statistics, the null hypothesis do not rejected,

$$
H_{0}=\theta_{1}=\theta_{2}=\theta_{3}=\theta_{4}=\theta_{5}
$$

$$
H_{a} \neq \theta_{1} \neq \theta_{2} \neq \theta_{3} \neq \theta_{4} \neq \theta_{4}
$$

However, the uses of several diagnostic tests were undertaken to check for the reliability and validity of this model.

\section{CCR, DOLS and FMOLS Estimators}

Using the Canonical Cointegration Regression (CCR), Dynamic OLS (DOLS), and Fully Modified OLS (FMOLS) approaches to confirm the long-run coefficients of PPI, GDP, REN, and FD in Equation (11). CCR (initiated by Park, 1992), FMOLS (initiated by Phillips and Hansen, 1990), and DOLS (initiated by Stock and Watson, 1993). DOLS and FMOLS allow asymptotic coherence by taking to account the effect of serial correlation. The normal distribution mixture is followed by CCR and FMOLS, which permit testing the asymptotic Chi-square, which overcomes the problem of a nonscalar nuisance.

\section{Gradual Shift Causality}

This study employed the Fourier Toda-Yamamoto causality test developed by Nazlioglu et al. (2016) to examine the causality between one variable (GDP, PPI, GDP, REN, FD) and another variable (EP). This technique allows structural breaks (gradual and smooths changes) in the analysis of causality. These breaks were taken into accounts using a Fourier Granger causality test with single frequency (SF) and cumulative frequencies (CF), respectively, known as Fourier approximation. The outcome of this technique is more reliable than the conventional causality test. Nazlioglu et al. (2016) built this model using the VAR $(p+d)$, which is defined as follows:

$$
y_{t}=\sigma(\mathrm{t})+\beta_{1} y_{t-1}+\cdots+\beta_{p+d \max } y_{t-(p+d \max )}+\varepsilon_{t}
$$




$$
\sigma(\mathrm{t})=\sigma_{0}+\sum_{k=1}^{x} \gamma_{1 k} \sin \left(\frac{2 \pi k t}{T}\right)+\sum_{k=1}^{x} \gamma_{2 k} \cos \left(\frac{2 \pi k t}{T}\right)
$$

303 where: the number of frequency was depicted as $\mathrm{x}, \gamma_{1 k}$ measures the size of the frequency while $\gamma_{2 k}$ measures changes 304 in frequency. Substitute Equation (15) in Equation (154), the Fourier Toda-Yamamoto causality with cumulative 305 frequencies $(\mathrm{CF})$ is defined as:

$$
y_{t}=\sigma_{0}+\sum_{k=1}^{n} \gamma_{1 k} \sin \left(\frac{2 \pi k t}{T}\right)+\sum_{k=1}^{n} \gamma_{2 k} \cos \left(\frac{2 \pi k t}{T}\right)+\beta_{1} y_{t-1}+\cdots+\beta_{p+d \max } y_{t-(p+d \max )}
$$

where: the approximation frequency was represented as k. Equation (17) defines the single-frequency components as:

$$
\sigma(\mathrm{t})=\sigma_{0}+\gamma_{1} \sin \left(\frac{2 \pi k t}{T}\right)+\gamma_{2} \cos \left(\frac{2 \pi k t}{T}\right)
$$

310 By substituting Equation (17) into Equation (14), the Fourier Toda-Yamamoto causality with single frequencies (SF) 311 is defined in Equation (14) as:

$$
y_{t}=\sigma_{0}+\gamma_{1} \sin \left(\frac{2 \pi k t}{T}\right)+\gamma_{2} \cos \left(\frac{2 \pi k t}{T}\right)+\beta_{1} y_{t-1}+\cdots+\beta_{p+d} y_{t-(p+d)}
$$

315 hypothesis of non-causality is zero $\left(\mathrm{H}_{0}: \beta_{1}=\beta_{\Theta}=0\right)$ against the alternate hypothesis $\left(\mathrm{H}_{0}: \beta_{1} \neq \beta_{\Theta} \neq 0\right)$.

\section{Finding and discussion}

Table 2 summarises the statistical features (a measure of central tendencies and dispersion) of the series. The considered variables are highly skewed $(-1<S>1)$, and the kurtosis of all series was platykurtic in nature except for PPI has a leptokurtic nature. All the series are normally distributed around the mean except PPI, as confirmed by the Jarque-Bera.

\begin{tabular}{|l|l|l|l|l|l|}
\hline \multicolumn{6}{|l|}{ Table 2: Descriptive Statistics } \\
\hline & EP & FD & GDP & REN & PPI \\
\hline Mean & $4.94 \mathrm{E}+08$ & 0.420911 & 9402.717 & 5273.958 & $5.89 \mathrm{E}+09$ \\
\hline Median & $4.96 \mathrm{E}+08$ & 0.421182 & 8803.741 & 5277.376 & $3.72 \mathrm{E}+09$ \\
\hline Maximum & $6.06 \mathrm{E}+08$ & 0.633827 & 11993.48 & 6855.265 & $2.97 \mathrm{E}+10$ \\
\hline Minimum & $3.62 \mathrm{E}+08$ & 0.160204 & 7442.972 & 3555.415 & 7300000. \\
\hline Std. Dev. & 65993353 & 0.156039 & 1404.898 & 1006.240 & $7.11 \mathrm{E}+09$ \\
\hline
\end{tabular}




\begin{tabular}{|l|l|l|l|l|l|}
\hline Skewness & -0.067958 & -0.122120 & 0.584863 & -0.052161 & 1.586153 \\
\hline Kurtosis & 2.287197 & 1.697691 & 1.904547 & 1.882748 & 5.486510 \\
\hline Jarque-Bera & 0.745961 & 2.487186 & 3.638393 & 1.783774 & 23.01552 \\
\hline Probability & 0.688679 & 0.288346 & 0.162156 & 0.409882 & 0.000010 \\
\hline Observations & 34 & 34 & 34 & 34 & 34 \\
\hline
\end{tabular}

323 Before exploring the co-integration among the variable used, it is important to verify the considered variables' 324 stationary characteristics. The problem of spurious regression is evident when the data are non-stationary (Granger 325 and Newbold, 1974) and to check whether any of the variables used are integrated at I(2). However, the ARDL restricts 326 the use of variables integrated at I(2). The conventional unit-roots (ADF and PP) were depicted in Table 3, whose 327 outcome reveals that no variable is integrated at $\mathrm{I}(2)$, but all the considered series are integrated at $\mathrm{I}(\mathrm{I})$. As mentioned 328 earlier, conventional unit-roots outcomes are often inconsistent because they do not incorporate the structural break(s) 329 during estimation. For this purpose, the study used the Zivot and Andrews (2002) unit root test, which was described 330 in Table 4. The outcome shows PPI is the only variable integrated at level, but all variables are integrated at I(I). 331 Hence, the considered variables are integrated at mixed order.

\begin{tabular}{|c|c|c|c|c|}
\hline \multicolumn{5}{|c|}{ Table 3: Unit Root without Break } \\
\hline & ADF & PP \\
\hline Variables & $\mathrm{I}(0)$ & $\mathrm{I}(\mathrm{I})$ & $\mathrm{I}(0)$ & $\mathrm{I}(\mathrm{I})$ \\
\hline EF & -2.8214 & $-5.5957^{*}$ & -2.7994 & $-5.5957^{*}$ \\
\hline GDP & -2.1667 & $-4.1198^{* *}$ & -1.8666 & $-4.0983^{* *}$ \\
\hline REN & -1.9865 & $-5.4813^{*}$ & -1.9865 & $5.4900^{*}$ \\
\hline PPI & -2.1280 & $-5.8105^{*}$ & -2.1334 & $-8.2278^{*}$ \\
\hline FD & -2.5490 & $-5.3930^{*}$ & -2.8193 & $-5.3930^{*}$ \\
\hline \multicolumn{4}{|c|}{$*$ and ** illustrates indicators $1 \%$ and $5 \%$ level significance } & \\
\hline
\end{tabular}

\begin{tabular}{|l|l|l|l|l|}
\hline \multicolumn{5}{|l|}{ Table 4: Unit Root with Break } \\
\hline & $\mathrm{I}(0)$ & $\mathrm{I}(\mathrm{I})$ \\
\hline Variables & T-statistics & Break-Year & T-statistics & Break-Year \\
\hline EF & -3.4287 & 2001 & $-7.2294^{*}$ & 2010 \\
\hline GDP & -3.7732 & 2010 & $-6.2933^{*}$ & 2010 \\
\hline REN & -2.4331 & 1991 & $-6.1372^{*}$ & 2003 \\
\hline PPI & $-6.1938^{*}$ & 1997 & $-8.0728^{*}$ & 2000 \\
\hline FD & -3.8038 & 1994 & $-7.8203^{*}$ & 2006 \\
\hline
\end{tabular}


Conventional co-integration techniques such as Johansen (1991) and Engle \& Granger (1987)) possess the inability to detect structural break, which can produce spurious analysis. For this purpose, the study employs the Maki (2012) co-integration, which captures at least five breaks in a co-integration model. The Maki co-integration test outcome was reported in Table 5. Table 5 reveals that at a 5\% significant level, co-integration association amongst ecological footprints and its regressors: PPI, GDP, REN, and FD at shift and trend regime over the considered period was evident. This asserts the presence of the co-movement among ecological footprints and its regressors in the presence of a structural break. The present study also used the bound testing approach as a robustness check for the

342 Maki co-integration outcome. As reported in Table 6, the null hypothesis was rejected at a 1\% significant level since 343 the F-statistics is greater than the critical value. It asserts the presence of the co-integration between ecological 344 footprint and its regressors.

\begin{tabular}{|l|l|l|l|}
\hline Table 5. Maki Co-integration Test \\
\hline & T-statistics & Critical Values & \\
\hline Model & \multicolumn{2}{|l|}{$5 \%$} & Break-Years \\
\hline \multicolumn{4}{|c|}{ Trend and Regime shifts } \\
\hline $\mathrm{EP}=f(\mathrm{GDP}$, PPI, FD, REN) & $-7.13909^{*}$ & -6.911 & 1998 \\
\hline $\mathrm{EP}=f(\mathrm{GDP}, \mathrm{PPI}, \mathrm{FD}, \mathrm{REN})$ & $-8.52593^{*}$ & -7.638 & 1998,1991 \\
\hline $\mathrm{EP}=f(\mathrm{GDP}, \mathrm{PPI}, \mathrm{FD}, \mathrm{REN})$ & $-10.2908^{*}$ & -8.254 & $1998,1991,2006$ \\
\hline $\mathrm{EP}=f(\mathrm{GDP}, \mathrm{PPI}, \mathrm{FD}, \mathrm{REN})$ & $-10.2908^{*}$ & -8.871 & $1998,1991,2006,2012$ \\
\hline $\mathrm{EP}=f(\mathrm{GDP}, \mathrm{PPI}, \mathrm{FD}, \mathrm{REN})$ & $-10.2908^{*}$ & -9.482 & $1998,1991,2006,2012,1995$ \\
\hline Note: $5 \%$ significance level is signified by $*$ & \\
\hline
\end{tabular}

\begin{tabular}{|c|c|c|c|}
\hline Table 5. ARDL bounds Test \\
\hline Model & Optimal Lag & F-Statistics & Co-integration \\
\hline EF $=f$ (GDP, REN, PPI, FD) & $(2,4,3,4,4)$ & $24.2468^{*}$ & Yes \\
\hline Level of Significance & L-BO & U-BO & \\
\hline $1 \%$ & 3.74 & 5.06 & \\
\hline $2.5 \%$ & 3.25 & 4.49 & \\
\hline $5 \%$ & 2.86 & 4.01 & \\
\hline $10 \%$ & 2.45 & 3.52 & \\
\hline \multicolumn{4}{|l|}{} \\
Note: $1 \%$ level of significance denoted as *. U-BO denotes the upper bound while L-BO \\
\hline
\end{tabular}


the short and long-run within the considered period. As described in Table 6, GDP, REN, PPI and FD are significant determinants of Brazil's ecological footprint for the short and long run. To be particular, we observed that GDP increases ecological footprint. It asserts that $1 \%$ GDP growth would increase ecological footprint by $1.33 \%$. This infers that economic growth contributes to environmental deterioration in Brazil. Since Brazil is an emerging economy that drives production and consumption considerably, which shows that Brazil is currently at the initial phase where growth is more important when compared to environmental quality, which provides credibility to the EKC hypothesis. This result demonstrates that environmental policy is ineffective at reducing ecological footprint triggered by higher levels of income. Kirikkaleli et al. 2020; Nathaniel et al. 2021; Hassan et al. 2019; Charfeddine \& Mrabet (2017); Wang \& Dong (2019); Bello et al.(2018); Destek \& Sinha (2020); Ahmed et al. (2020); Alola et al. 2019; Dogan et al. (2019) agree with the finding. In the short run, GDP also contributes to environmental degradation since the interaction between GDP and the ecological footprint is positive. The interaction between renewable energy use and ecological footprints is negative and statistically significant. This shows that ecological footprints will decrease by $0.46 \%$ will be caused by a $1 \%$ increase in renewable energy use. As expected, the use of renewable energy improves environmental quality. This outcome's possible cause is that Brazil has pursued a clean energy matrix policy and has established incentives for more low-carbon measures (Pereira et al., 2012). About 47\% of Brazil's energy mix are renewable energy sources, with $89 \%$ of the electricity supplied are from renewable energy resources (IEA, 2020).

After China and the United States, Brazil is the world's third-largest user of renewable sources (Pao et al., 2013). This indicates that Brazil is on the path of attaining SDGs through advancements in renewable energy technologies. This aligns with the study of Wang \& Dong (2019), Usman et al. (2021), Murshed et al. (2021), Ulucak et al. (2020), Destek \& Sinha (2020), and Alola et al. (2019) for 14 sub-Saharan African nations, , 15 highest emitting countries, South Asia, BRICS, OECD countries and EU countries respectively. A similar outcome was established between ecological footprint and renewable energy use in the short run. As against our expectation, public-private partnership investment in energy has a positive effect on ecological footprint. It infers that a $1 \%$ increase in the PPI will lead to the rise of the ecological footprint by $0.019 \%$. This implication of this outcome means that PPI contributes to environmental degradation. A reasonable explanation for this outcome could be a strong connection between PPI and energy sources related to fossil fuel. The study of Kirikkaleli \& Adebayo (2021), Shahbaz et al. (2020), and Khan, et al. (2020) are consistent with this outcome. In the short run, a similar outcome was established between PPI and ecological footprint. The interconnection between financial development and ecological footprint is negative and significant. This implies financial development reduces environmental deterioration.

Increasing financial development by $1 \%$ triggers a decrease in environmental degradation by $0.17 \%$. This negative connection affirms that financial services have reached a stage where funds or resources can be diverted to environmentally friendly initiatives. The use of the financial sector can be a strategic and effective instrument in mitigating environmental degradation. Similar studies such as Baloch et al. (2019), Naqvi et al. (2020), and Destek \& Sarkodie (2019) also corroborate this outcome, but Usman et al. (2020a) outcome for the USA is inconsistent with this finding. Finally, disequilibrium is evident in the short run, but the correction is made at the speed of $41 \%$, the 
385 footprint and its regressors is corrected, indicating convergence towards the equilibrium in the long run. Diagnostic 386 tests were carried out was also reported in Table 6 and Figure 1 and 2.

\begin{tabular}{|c|c|c|c|c|c|}
\hline \multicolumn{6}{|c|}{ Table 6: ARDL estimates } \\
\hline Dependent Variable & Analysis & Regressors & Coefficient & Error term & T-statistics \\
\hline \multirow[t]{4}{*}{ EP } & \multirow[t]{4}{*}{ Long run } & GDP & 1.3336 & 0.1390 & $9.5918 *$ \\
\hline & & REN & -0.4635 & 0.0914 & $-5.0711 *$ \\
\hline & & PPI & 0.0196 & 0.0033 & $5.8629^{*}$ \\
\hline & & FD & -0.1786 & 0.0568 & $-3.4599^{*}$ \\
\hline \multirow[t]{5}{*}{ EP } & \multirow[t]{5}{*}{ Short-run } & ECM(-) & -0.4117 & 0.0305 & $-13.4852 *$ \\
\hline & & GDP & 1.3336 & 0.0679 & $19.6138^{*}$ \\
\hline & & REN & -0.1153 & 0.0419 & $-2.7517 * *$ \\
\hline & & PPI & 0.01963 & 0.0019 & $10.3117^{*}$ \\
\hline & & FD & -0.1786 & 0.0259 & $8.2036^{*}$ \\
\hline \multicolumn{6}{|l|}{ Diagnostic Tests } \\
\hline$\chi^{2}$ Heteroscedasticity & $0.7893(0.6880)$ & & & & \\
\hline$\chi^{2}$ Ramsey & $4.3136(0.9949)$ & & & & \\
\hline$\chi^{2}$ Normality & $0.9610(0.6184)$ & & & & \\
\hline$\chi^{2} \mathrm{LM}$ & $2.0493(0.2097)$ & & & & \\
\hline
\end{tabular}

387

10.0

7.5

5.0

2.5

0.0

$-2.5$

$-5.0$

$-7.5$

$-10.0$

$\begin{array}{llllllll}2010 & 2011 & 2012 & 2013 & 2014 & 2015 & 2016 & 2017\end{array}$ 


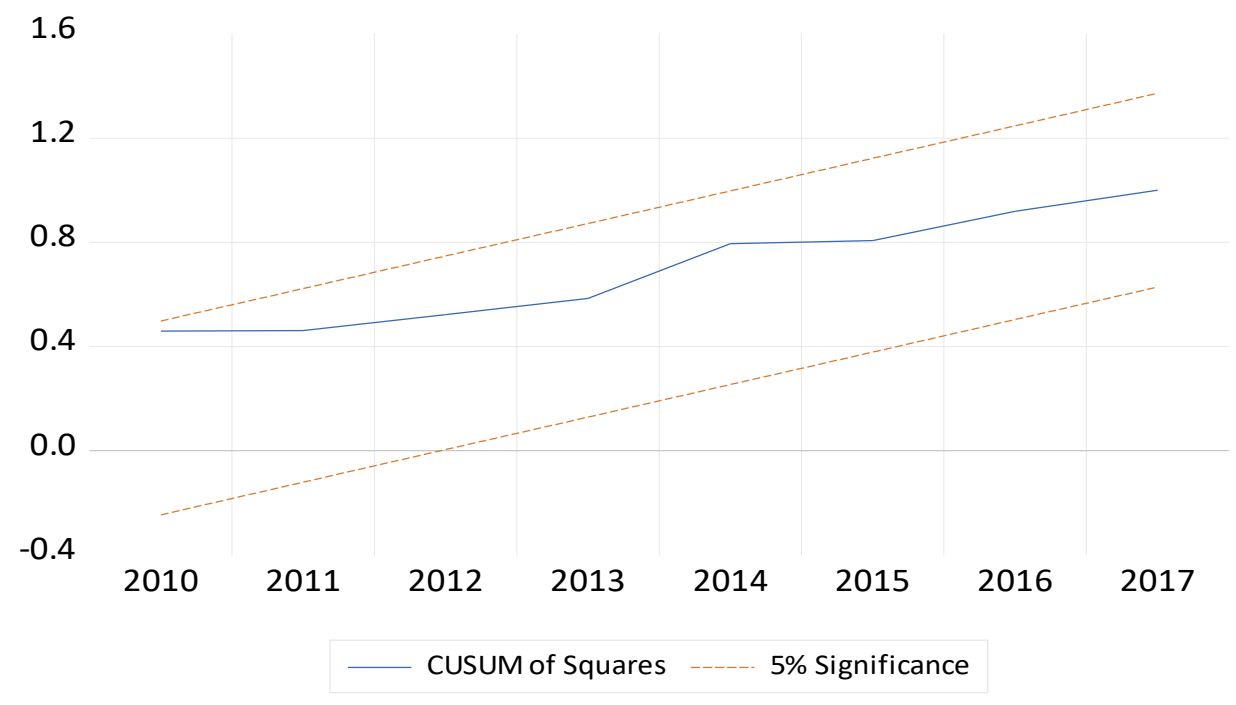

Figure 2: CUSUMSQ

The robustness check of the long-run estimate was examined, utilizing the CCR, DOLS, and FMOLS methods. The outcome of the CCR, DOLS and FMOLS methods is reported in Table 7. The long-run elasticities for CCR, DOLS and FMOLS is seen to corroborate with ARDL estimates in the long. To be particular, the FMOLS method reveals the long-run elasticities of GDP, REN, PPI and FD are $1.32 \%,-0.12 \%, 0.02 \%$, and $-0.18 \%$, respectively. Using the DOLS, the long-run elasticities of GDP, REN, PPI, and FD are $1.33 \%,-0.12 \%, 0.02 \%$, and $0.18 \%$, respectively. Estimating the model using CRR, the long-run elasticities of GDP, REN, PPI, and FD are 1.29\%, $-0.34 \%, 0.03 \%$, and $-0.31 \%$. We observed a similar outcome was evident in FMOLS and DOLS outcome but the estimates from CRR is slightly higher.

\begin{tabular}{|c|c|c|c|c|c|c|}
\hline \multicolumn{2}{|c|}{ Table 7: Robustness Check for ARDL Coefficients } \\
\hline & \multicolumn{2}{|c|}{ FMOLS } & \multicolumn{2}{c|}{ DOLS } & \multicolumn{2}{c|}{ CRR } \\
\hline Regressors & Coefficient & T-statistic & Coefficient & T-statistic & Coefficient & T-statistic \\
\hline GDP & 1.3273 & $29.7859^{*}$ & 1.3336 & $20.0340^{*}$ & 1.2922 & $8.9173^{*}$ \\
\hline REN & -0.1189 & $-3.2852^{*}$ & -0.1153 & $-2.1351^{* *}$ & -0.3375 & $-4.1344^{*}$ \\
\hline PPI & 0.0197 & $11.7032^{*}$ & 0.0196 & $9.0341^{*}$ & 0.0341 & $8.7558^{*}$ \\
\hline FD & -0.1810 & $-13.9429^{*}$ & -0.1786 & $-7.8084^{*}$ & -0.3052 & $-6.9741^{*}$ \\
\hline$* \& * *$ stand for $1 \% \& 10 \%$ significance level correspondingly & \\
\hline
\end{tabular}

It is not sufficient to determine whether the chosen variables have a positive or negative relationship; it is, therefore, necessary to determine which variable is influencing or transmitting directly to the other variable. This helps policymakers to formulate policies that is necessary for adjustments. In exposing this flowing (Uni-directional or Bidirectional) among the considered variable, the Granger causality provides solid ground for this. Since conventional 
estimation, this study utilizes the Gradual Shift Causality test to detect the causality between dependent variable i.e ecological footprint and its regressors i.e. GDP, PPI, REN and FD, was reported in Table 8. The novelty of the Gradual shift causality has to do with examining the causality between series in the presence of structural break. We discover from Table 8 that there is a feedback causal association between GDP and EP, indicating the change in EP will significantly lead to GDP and GDP causes EP in Brazil. Since Brazil is an industrialized economy whose economic development is accelerated by the exploitation and extraction of natural resources causes a reduction in the environment biocapacity while the ecological footprint increases. However, with the integration of sustainability management into consumption and production, the pace of natural resource depletion and environmental stress reduction enables resources to be regenerated (United Nations, 2015). This outcome is consistent with Ibrahiem \& Hanafy (2020), Danish \& Khan (2019), Destek \& Sarkodie (2019) for Egypt, Brics economies, 11 newly industrialized economies, respectively.

The unidirectional causal association was found from renewable energy to ecological footprint, which implies that renewable energy has predictive control over the ecological footprint. This suggests that any green energy policy has a considerable impact on ecological footprint. This result aligns with previous work done by Naqvi et al. (2020). We detected a unidirectional causal interaction from ecological footprint to public-private partnership investment. This indicates that ecological footprint is a predicting factor of public-private partnership investment. The bidirectional causal association was evident between financial development and ecological footprint, establishing a feedback hypothesis. The variation in ecological footprint will explain financial development in the future and vice versa. This is consistent with the findings reported by Chen et al. (2019), Usman \& Hammar (2020), and Yao et al. (2021).

\begin{tabular}{|c|c|c|c|c|}
\hline \multicolumn{5}{|c|}{ Table 8: Gradual Shift Causality Test } \\
\hline Causality Path & Wald-stat & No of Fourier & P-Value & Decision \\
\hline $\mathrm{GDP} \rightarrow \mathrm{EP}$ & $32.947 *$ & 2 & 0.000 & Reject Ho \\
\hline $\mathrm{EP} \rightarrow \mathrm{GDP}$ & $13.305^{*}$ & 2 & 0.0650 & Reject Ho \\
\hline $\mathrm{EP} \rightarrow \mathrm{REN}$ & 4.0920 & 1 & 0.7691 & Do not Reject Ho \\
\hline $\mathrm{REN} \rightarrow \mathrm{EP}$ & $31.130^{*}$ & 1 & 0.0000 & Reject Ho \\
\hline $\mathrm{EP} \rightarrow \mathrm{PPI}$ & $14.844 * *$ & 3 & 0.0380 & Reject Ho \\
\hline $\mathrm{PPI} \rightarrow \mathrm{EP}$ & 9.7492 & 3 & 0.2032 & Do not Reject Ho \\
\hline $\mathrm{EP} \rightarrow \mathrm{FD}$ & $24.672 *$ & 3 & 0.0008 & Reject Ho \\
\hline $\mathrm{FD} \rightarrow \mathrm{EP}$ & $23.889 *$ & 3 & 0.0011 & Reject Ho \\
\hline
\end{tabular}

\section{Conclusion and policy implication}

This research explores the long-term and causal impact of economic growth and renewable energy on ecological footprint by considering the role of public-private partnership investment in energy and financial development for 
have investigated this association in the context of Brazil. Both the Maki and ARDL bound testing co-integration techniques were used to explore the long-run co-integration. These co-integration techniques confirm co-integration was evident amongst PPI, REN, FD, GDP, and EP. For the long-run coefficient, we capitalized on the following estimators: ARDL, FMOLS, DOLS, and CRR. These estimators' finding reveals that GDP and PPI have a positive and significant influence on ecological footprint; financial development and renewable energy have a detrimental impact on Brazil's ecological footprint. Furthermore, the causal direction was detected using the Gradual- Shift based on Fourier approximation. This finding showed two bidirectional causality associations between EP and GDP, EP and FD but one-way causality from EP to PPI and from REN to EP in Brazil.

This study provides policy ideas that give policymakers and environmentalists valuable perspectives. Fossil fuel in Brazil is arguably more than renewable energy sources. For this purpose, renewable energy resources should be increased in Brazil's energy mix to a significant proportion to reduce environmental degradation. The government can achieve it by increasing incentives for low carbon energy use and tax exemption for companies and households using clean technologies. Furthermore, investing in renewable energy should be promoted through public-private partnerships by developing a juicy policy for both parties. The establishment of a forum that will foster public and private partnerships will enhance communication, which will create collaboration for new initiatives for green technological innovations. The government can assist the financial market by formulating a framework that would promote low carbon technology development. Industrialists in Brazil need to be encouraged to use the financial sectors' facilities to invest in research and development of efficient, eco-friendly technologies for production; it will help significantly achieve SDGs 7 and 13. Finally, sustaining growth in the economy is obligatory but under the constraint of safeguarding the environment. In this context, no single and clear policy would mitigate environmental pressures without impeding sustainable growth. However, adopting policies that will reduce environmental degradation. In this study, we establish an association utilizing recent econometric techniques; there are certain limitations. The timeframe of data (1983-2017) is one of the limitations of this study. Thus, future studies could concentrate on examining the economic complexity of environmental pollution by taking into account structural break(s). Finally, the impact on public-private investments in energy on ecological footprint could be investigated utilizing recent data with another determinant of environmental degradation.

\section{Ethical Approval}

We confirmed that this manuscript has not been published elsewhere and is not under consideration by another journal. Ethical approval and Informed consent do not applicable for this study.

\section{Consent to Participate}

Not applicable

\section{Consent to Publish}

Not applicable

\section{Authors Contributions}

Tomiwa Sunday Adebayo, Dervis Kirikkaleli and Gbenga Daniel Akinsoladesigned the experiment and collect the dataset. The introduction and literature review sections are written by Sukru Umarbeyli, Abraham Ayobamiji Awosusi 
and Ibrahim Adeshola. Dervis Kirikkaleli and Gbenga Daniel Akinsola constructed the methodology section and empirical outcomes in the study. Gbenga Daniel Akinsola, Tomiwa Sunday Adebayo and Sukru Umarbeyli contributed to the interpretation of the outcomes. All the authors read and approved the final manuscript.

473

\section{Funding}

We also confirmed that this research did not receive any specific grant from funding agencies in the public, commercial, or not-for-profit sectors.

\section{Competing Interests}

The authors declares no competing interests.

\section{Availability of data and materials}

The data that support the findings of this study are available from the World Bank.

\section{References}

Adebayo, T. S., Awosusi, A. A., \& Eminer, F. (2020). Stock market-growth relationship in an emerging economy: empirical finding from ARDL-based bounds and causality approaches. Journal of Economics and Business, 3(2).

Adebayo, T. S., \& Akinsola, G. D. (2021). Investigating the causal linkage among economic growth, energy consumption and $\mathrm{CO} 2$ emissions in Thailand: an application of the wavelet coherence approach. Int J Renew Energy Dev, 10(1), 17-26.

Ahmed, Z., Wang, Z., Mahmood, F., Hafeez, M., \& Ali, N. (2019). Does globalization increase the ecological footprint? Empirical evidence from Malaysia. Environmental Science and Pollution Research, 26(18), 18565-18582.

Ahmed, Z., Asghar, M. M., Malik, M. N., \& Nawaz, K. (2020). Moving towards a sustainable environment: the dynamic linkage between natural resources, human capital, urbanization, economic growth, and ecological footprint in China. Resources Policy, 67, 101677.

Ahmed, Z., Zhang, B., \& Cary, M. (2021). Linking economic globalization, economic growth, financial development, and ecological footprint: Evidence from symmetric and asymmetric ARDL. Ecological Indicators, 121, 107060 .

Alola, A. A., Bekun, F. V., \& Sarkodie, S. A. (2019). Dynamic impact of trade policy, economic growth, fertility rate, renewable and non-renewable energy consumption on ecological footprint in Europe. Science of the Total Environment, 685, 702-709.

Ansari, M. A., Ahmad, M. R., Siddique, S., \& Mansoor, K. (2020). An environment Kuznets curve for ecological footprint: Evidence from GCC countries. Carbon Management, 11(4), 355-368.

Ayobamiji, A. A., \& Kalmaz, D. B. (2020). Reinvestigating the determinants of environmental degradation in Nigeria. International Journal of Economic Policy in Emerging Economies, 13(1), 52-71. 
Baloch, M. A., Zhang, J., Iqbal, K., \& Iqbal, Z. (2019). The effect of financial development on ecological footprint in BRI countries: evidence from panel data estimation. Environmental Science and Pollution Research, 26(6), 6199-6208.

Bello, M. O., Solarin, S. A., \& Yen, Y. Y. (2018). The impact of electricity consumption on CO2 emission, carbon footprint, water footprint and ecological footprint: the role of hydropower in an emerging economy. Journal

BP Statistical Review of World Energy, (2020). https://www.bp.com/content/dam/bp/businessof environmental management, 219, 218-230.

Charfeddine, L. (2017). The impact of energy consumption and economic development on ecological footprint and $\mathrm{CO} 2$ emissions: evidence from a Markov switching equilibrium correction model. Energy Economics, 65, 355-374.

Charfeddine, L., \& Mrabet, Z. (2017). The impact of economic development and social-political factors on ecological footprint: A panel data analysis for 15 MENA countries. Renewable and Sustainable Energy Reviews, 76, $138-154$.

Chen, S., Saud, S., Saleem, N., \& Bari, M. W. (2019). Nexus between financial development, energy consumption, income level, and ecological footprint in CEE countries: do human capital and biocapacity matter?. Environmental Science and Pollution Research, 26(31), 31856-31872.

Danish, U. R., \& Khan, S. U. D. (2019). Determinants of the ecological footprint: Role of renewable energy, natural resources, and urbanization. Sustain Cities Soc 54: 101996.

Destek, M. A., \& Sarkodie, S. A. (2019). Investigation of environmental Kuznets curve for ecological footprint: the role of energy and financial development. Science of the Total Environment, 650, 2483-2489.

Destek, M. A., \& Sinha, A. (2020). Renewable, non-renewable energy consumption, economic growth, trade openness and ecological footprint: Evidence from organisation for economic Co-operation and development countries. Journal of Cleaner Production, 242, 118537.

533 Dogan, E., Taspinar, N., \& Gokmenoglu, K. K. (2019). Determinants of ecological footprint in MINT countries. Energy \& Environment, 30(6), 1065-1086.

535 Engle, R. F., \& Granger, C. W. (1987). Co-integration and error correction: representation, estimation, and testing. Econometrica: journal of the Econometric Society, 251-276. 
Godil, D. I., Sharif, A., Rafique, S., \& Jermsittiparsert, K. (2020). The asymmetric effect of tourism, financial development, and globalization on ecological footprint in Turkey. Environmental Science and Pollution Research, 27(32), 40109-40120.

Granger, C. W., Newbold, P., \& Econom, J. (1974). Spurious regressions in econometrics. Baltagi, Badi H. A Companion of Theoretical Econometrics, 557-61.

Global Ecological footprint (2021) Retrieved from https://api.footprintnetwork.org/v1/data/21/all/BCtot,EFCtot. Accessed March 2021

Hassan, S. T., Xia, E., Khan, N. H., \& Shah, S. M. A. (2019). Economic growth, natural resources, and ecological footprints: evidence from Pakistan. Environmental Science and Pollution Research, 26(3), 2929-2938.

International Monetary Fund (IMF) (2020) Retrieved from imf.org/en/Data. Accessed March 2021

Johansen, S. (1991). Estimation and hypothesis testing of co-integration vectors in Gaussian vector autoregressive models. Econometrica: journal of the Econometric Society, 1551-1580.

Kassouri, Y., \& Altıntaş, H. (2020). Human well-being versus ecological footprint in MENA countries: A tradeoff?. Journal of environmental management, 263, 110405.

Khan, Z., Ali, M., Kirikkaleli, D., Wahab, S., \& Jiao, Z. (2020). The impact of technological innovation and publicprivate partnership investment on sustainable environment in China: Consumption-based carbon emissions analysis. Sustainable Development, 28(5), 1317-1330.

Khan, I., Hou, F., \& Le, H. P. (2021). The impact of natural resources, energy consumption, and population growth on environmental quality: Fresh evidence from the United States of America. Science of the Total Environment, 754, 142222.

Kirikkaleli, D., \& Adebayo, T. S. (2021). Do public-private partnerships in energy and renewable energy consumption matter for consumption-based carbon dioxide emissions in India?. Environmental Science and Pollution Research, 1-14.

Kirikkaleli, D., Adebayo, T. S., Khan, Z., \& Ali, S. (2020). Does globalization matter for ecological footprint in Turkey? Evidence from dual adjustment approach. Environmental Science and Pollution Research, 1-9.

Langnel, Z., \& Amegavi, G. B. (2020). Globalization, electricity consumption and ecological footprint: An autoregressive distributive lag (ARDL) approach. Sustainable Cities and Society, 63, 102482. consumption, capital formation, globalization, $\mathrm{CO} 2$ emissions and economic growth nexus in Malaysia: Fresh evidence from combined co-integration and causality analysis. Energy Strategy Reviews, 31, 100526. 
Murshed, M., Haseeb, M., \& Alam, M. S. (2021). The Environmental Kuznets Curve hypothesis for carbon and ecological footprints in South Asia: The role of renewable energy. GeoJournal, 1-28.

Nathaniel, S., \& Khan, S. A. R. (2020). The nexus between urbanization, renewable energy, trade, and ecological footprint in ASEAN countries. Journal of Cleaner Production, 272, 122709.

Nathaniel, S. P., Murshed, M., \& Bassim, M. (2021). The nexus between economic growth, energy use, international trade and ecological footprints: The role of environmental regulations in N11 countries. Energy, Ecology and Environment, 1-17.

Nathaniel, S., Nwodo, O., Adediran, A., Sharma, G., Shah, M., \& Adeleye, N. (2019). Ecological footprint, urbanization, and energy consumption in South Africa: including the excluded. Environmental Science and Pollution Research, 26(26), 27168-27179.

Nazlioglu, S., Gormus, N. A., \& Soytas, U. (2016). Oil prices and real estate investment trusts (REITs): Gradual-shift causality and volatility transmission analysis. Energy Economics, 60, 168-175.

Naqvi, S. A. A., Shah, S. A. R., \& Mehdi, M. A. (2020). Revealing empirical association among ecological footprints, renewable energy consumption, real income, and financial development: a global perspective. Environmental Science and Pollution Research, 27(34), 42830-42849.

Pao, H. T., \& Fu, H. C. (2013). Renewable energy, non-renewable energy and economic growth in Brazil. Renewable and Sustainable Energy Reviews, 25, 381-392.

Park, J. Y. (1992). Canonical cointegrating regressions. Econometrica: Journal of the Econometric Society, 119-143.

Pata, U. K., \& Yilanci, V. (2020). Financial development, globalization and ecological footprint in G7: further evidence from threshold co-integration and fractional frequency causality tests. Environmental and Ecological Statistics, 27(4), 803-825.

Pereira, M. G., Camacho, C. F., Freitas, M. A. V., \& Da Silva, N. F. (2012). The renewable energy market in Brazil:

Pesaran, M. H., Shin, Y., \& Smith, R. J. (2001). Bounds testing approaches to the analysis of level

Phillips, P. C., \& Hansen, B. E. (1990). Statistical inference in instrumental variables regression with I (1) processes. The Review of Economic Studies, 57(1), 99-125.

594 Phillips, P. C., \& Perron, P. (1988). Testing for a unit root in time series regression. Biometrika, 75(2), 335-346. 
Rjoub, H., Odugbesan, J. A., Adebayo, T. S., \& Wong, W. K. (2021). Sustainability of the Moderating Role of Financial Development in the Determinants of Environmental Degradation: Evidence from Turkey. Sustainability 2021, 13, 1844.

Rudolph, A., \& Figge, L. (2017). Determinants of ecological footprints: what is the role of globalization?. Ecological Indicators, $81,348-361$.

Sabir, S., \& Gorus, M. S. (2019). The impact of globalization on ecological footprint: empirical evidence from the South Asian countries. Environmental Science and Pollution Research, 26(32), 33387-33398.

Said, S. E., \& Dickey, D. A. (1984). Testing for unit roots in autoregressive-moving average models of unknown order. Biometrika, $71(3)$, 599-607.

Saud, S., Chen, S., \& Haseeb, A. (2020). The role of financial development and globalization in the environment: Accounting ecological footprint indicators for selected one-belt-one-road initiative countries. Journal of Cleaner Production, 250, 119518.

Shahbaz, M., Nasir, M. A., \& Roubaud, D. (2018). Environmental degradation in France: the effects of FDI, financial development, and energy innovations. Energy Economics, 74, 843-857.

Shahbaz, M., Raghutla, C., Song, M., Zameer, H., \& Jiao, Z. (2020). Public-private partnerships investment in energy as new determinant of $\mathrm{CO} 2$ emissions: the role of technological innovations in China. Energy Economics, 86, 104664.

Stock, J. H., \& Watson, M. W. (1993). A simple estimator of cointegrating vectors in higher order integrated systems. Econometrica: Journal of the Econometric Society, 783-820.

614 Udemba, E. N. (2020). A sustainable study of economic growth and development amidst ecological footprint: New insight from Nigerian Perspective. Science of the Total Environment, 732, 139270.

616 Ulucak, R., \& Khan, S. U. D. (2020). Determinants of the ecological footprint: role of renewable energy, natural resources, and urbanization. Sustainable Cities and Society, 54, 101996.

618 Usman, M., \& Hammar, N. (2020). Dynamic relationship between technological innovations, financial development, renewable energy, and ecological footprint: fresh insights based on the STIRPAT model for Asia Pacific Economic Cooperation countries. Environmental Science and Pollution Research, 1-18.

Usman, O., Akadiri, S. S., \& Adeshola, I. (2020a). Role of renewable energy and globalization on ecological footprint in the USA: implications for environmental sustainability. Environmental Science and Pollution Research, 27, 30681-30693. 
United Nations (2015) Sustainable development goals. Retrieved from https://sdgs.un.org/goals Accessed on 5, March 2021.

626 Usman, O., Iortile, I. B., \& Ike, G. N. (2020b). Enhancing sustainable electricity consumption in a large ecological reserve-based country: the role of democracy, ecological footprint, economic growth, and globalisation in Brazil. Environmental Science and Pollution Research, 27(12), 13370-13383.

Usman, M., Makhdum, M. S. A., \& Kousar, R. (2021). Does financial inclusion, renewable and non-renewable energy utilization accelerate ecological footprints and economic growth? Fresh evidence from 15 highest emitting countries. Sustainable Cities and Society, 65, 102590.

Wang, Z., Bui, Q., Zhang, B., \& Pham, T. L. H. (2020). Biomass energy production and its impacts on the ecological footprint: An investigation of the G7 countries. Science of the Total Environment, 743, 140741.

634 Wang, J., \& Dong, K. (2019). What drives environmental degradation? Evidence from 14 Sub-Saharan African countries. Science of the Total Environment, 656, 165-173.

Waqih, M. A. U., Bhutto, N. A., Ghumro, N. H., Kumar, S., \& Salam, M. A. (2019). Rising environmental degradation and impact of foreign direct investment: an empirical evidence from SAARC region. Journal of environmental management, 243, 472-480.

639 World Bank (2021) World development indicators. http://data.worldbank.org/. Accessed March 2021

640 Yao, X., Yasmeen, R., Hussain, J., \& Shah, W. U. H. (2021). The repercussions of financial development and corruption on energy efficiency and ecological footprint: Evidence from BRICS and Next 11 Countries. Energy, 120063.

643 Yilanci, V., \& Pata, U. K. (2020). Investigating the EKC hypothesis for China: the role of economic complexity on 644 ecological footprint. Environmental Science and Pollution Research, 1-12.

645 Zivot, E., \& Andrews, D. W. K. (2002). Further evidence on the great crash, the oil-price shock, and the unit-root hypothesis. Journal of business \& economic statistics, 20(1), 25-44. 
Figures

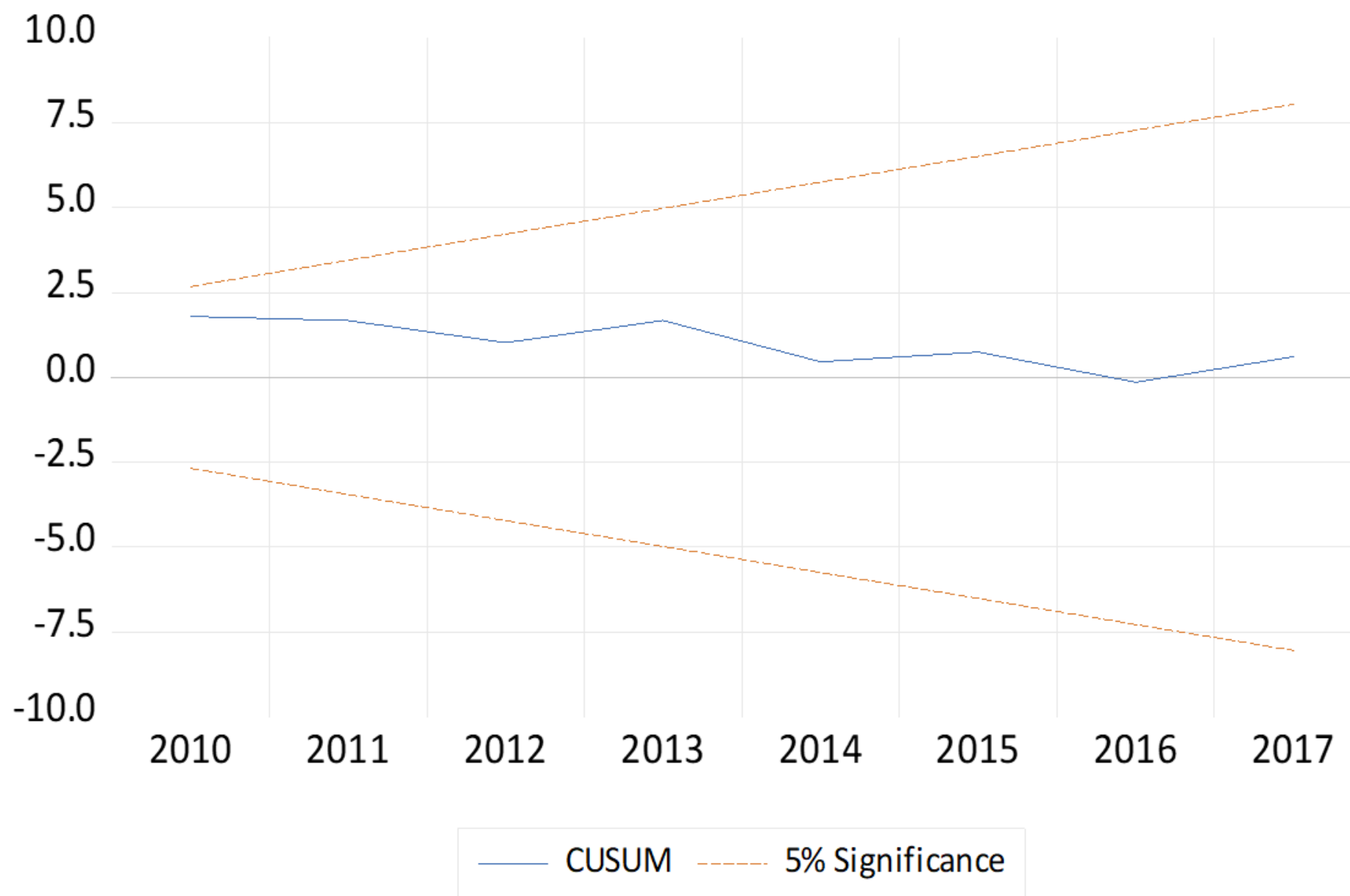

Figure 1

CUSUM 


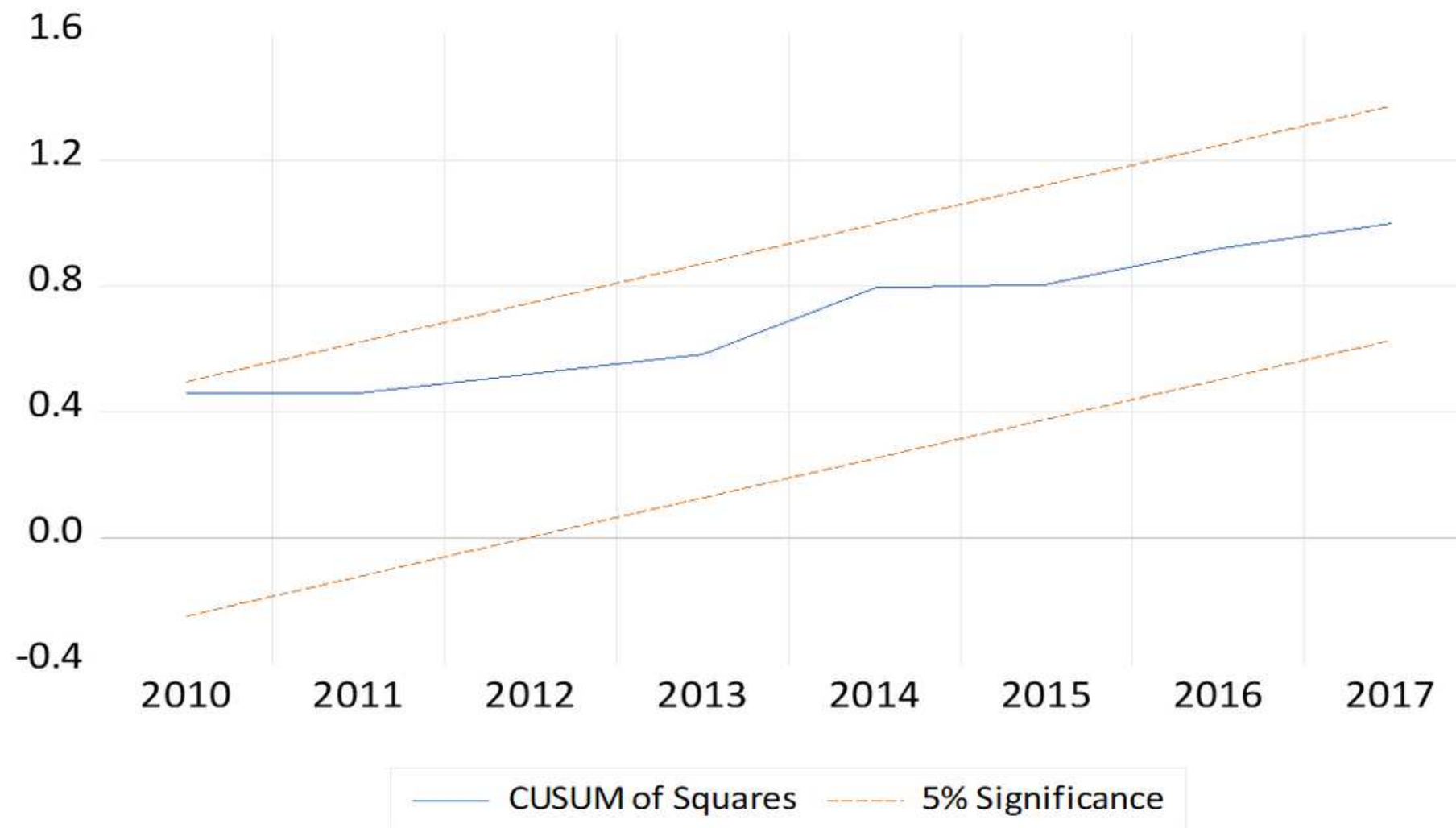

Figure 2

CUSUMSQ 